\title{
LA PLANEACIÓN ESTRATÉGICA COMO HERRAMIENTA EN LAS EMPRESAS
}

\author{
AUTORES: José Peñafiel Loor ${ }^{1}$ \\ Lucy Yulexi Muñiz Soledispa ${ }^{2}$ \\ Luis Fernando Mosquera Tuárez ${ }^{3}$
}

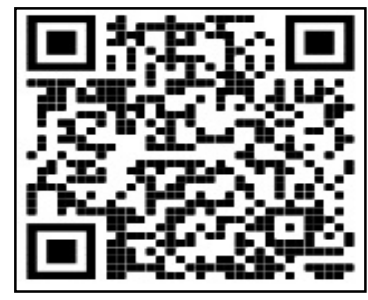

DIRECCIÓN PARA CORRESPONDENCIA: jpfelix@hotmail.es

Fecha de recepción: 25/10/2020

Fecha de aceptación: 28/11/2020

\section{RESUMEN}

El presente artículo trata de ostentar la importancia de la planeación estratégica como herramienta en las organizaciones. Por lo cual se planteó como objetivos de estudio determinar la importancia de contar con una planeación estratégica como herramienta en las organizaciones. Comprender la función que cumple la planificación estratégica dentro de las organizaciones. Estudiar los distintos modelos de planeación estratégica más conocidos. Determinar cuáles son las ventajas y desventajas de la planificación estratégica. Al hablar de planeación estratégica es dar a entender en términos generales y de la manera en la que se determinarán ostentaciones de comportamiento que necesariamente van a incidir de forma profunda y explicativa en el expectante de la compañía u entidad que la adopta. Hoy en día se piensa en la empresa como fracción de un entorno y en métodos de opciones o alternativas en función de lo que se posee, de lo que la rodea y de las distintas oportunidades y horizontes posibles que pueden llevar a conseguir el objetivo establecido. La planeación estratégica tiene distintos beneficios que exceden a sus desventajas, si es aplicada de manera correcta, no obstante, sufre riesgos inherentes que pueden ser prevalecidos con la vigilancia e intervención.

PALABRAS CLAVE: Planificación; Administración; Competitividad; Oportunidad; Modelos.

\section{STRATEGIC PLANNING AS A TOOL IN COMPANIES}

\section{ABSTRACT}

This article tries to show the importance of strategic planning as a tool in organizations. Therefore, it was proposed as study objectives determine the importance of having strategic planning as a tool in organizations. Understand the role of strategic planning within organizations. Study the different best-known strategic planning models. Determine what are the advantages and disadvantages of strategic planning. When talking about strategic planning is to imply in general terms and the way in which behavioral ostentations will be determined that will necessarily have a profound and explanatory impact on the expectant of the company or entity that adopts it. Nowadays, the company is thought of as a fraction of an environment and of

\footnotetext{
${ }^{1}$ Docente, Universidad Estatal Del Sur De Manabí, Jipijapa, Ecuador. E-mail: jpfelix@hotmail.es

${ }^{2}$ Estudiante, Universidad Estatal Del Sur De Manabí, Jipijapa, Ecuador. E-mail: yulexisoledispa@gmail.com

${ }^{3}$ Estudiante, Universidad Estatal Del Sur De Manabí, Jipijapa, Ecuador. E-mail: fernando.97scorpion@gmail.com
} 
José Peñafiel Loor, Lucy Yulexi Muñiz Soledispa, Luis Fernando Mosquera Tuárez

methods of options or alternatives depending on what it possesses, what surrounds it and the different opportunities or possible horizons that can lead to achieving the established objective. Strategic planning has different benefits that exceed its disadvantages, if applied correctly; however, it suffers from inherent risks that may be prevalent with surveillance and intervention.

KEYWORDS: Planning; Administration; Competitiveness; Opportunity; Models.

\section{INTRODUCCIÓN}

El hábitat de los negocios a nivel global se ha visto afectado a lo extendido de la historia por diversos horizontes, desde entonces la administración y la gestión hayan desarrollado rumbos cronológicos cuyo fin de hacer frente a la complejidad, el comienzo y la incapacidad de la predicción. A medida que se ha ido creando más arduo, sugestivo y menos imaginable. Los distintos sistemas se han hecho más sofisticados y las distintas novedades se han asemejando a las anteriores. De esa manera el avance de la gestión y la administración inciden en una réplica de ajuste al futuro descendiente.

Las empresas y un porcentaje de las organizaciones, están en competitividad en los elementos productivos, sus ingresos, los clientes, con el que hacer frente a los precios. Los administradores asumen el arduo compromiso en la toma de decisiones para llevar el diseño de la estrategia empresarial dentro de las organizaciones, entre estas tenemos la elección de productos y servicios que brindar, el diseño y la ejecución de las políticas que establecen como instituir los enfoques competitivos de la compañía en el mercado. Es trascendental entonces efectuar una planeación estratégica de las empresas para que logren alcanzar la ventaja competitiva anhelada. Dentro de una Planeación Estratégica se debe fijar que debe crear, cómo se va a crear y cómo puede darse cuenta una empresa si efectúa o no con los objetivos esperados y determinados.

Según el autor (Rovere, 2006), la planeación estratégica está vinculada de manera inédita con el proceso total de la dirección, por lo que cualquier empresa que no cuente con algún tipo de formalidad en su sistema de planeación corre el riesgo de que suceda un siniestro ineludible. El presente artículo enseña la conceptualización de la estrategia, formulación, sus componentes, las características de la planeación los beneficios, sus limitaciones, y su aplicación como una herramienta en las organizaciones.

La investigación efectuada es de tipo explicativa y cualitativa encaminada a una agrupación de datos publicados en revistas científicas que accedan un mejor entendimiento de los significados en el tema a tratar, basado en la inducción investigativa de la información obtenida. Desde este enfoque de la investigación propuesta, se inició con la recopilación de los artículos que contuvieran el término "planeación estratégica”, en la base de datos de masscience.com.

El uso de esta base de información se realizó teniendo en cuenta que esta cuenta con varias revistas de profundo impacto, lo cual abre paso a una información relevante razonablemente fiable y valida, la misma que ha sido analizada por varios autores eruditos en el tema que a su vez esta basadas bajo estándares internacionales de arbitrajes reconocidos y acreditados que ayudan a referirse a estas bases científicas. 
Acorde con lo anterior se logró obtener un total de 50 publicaciones se tomaron las 9 más relevantes aprovechadas para su análisis en comparación con los objetivos de esta investigación. Además, la indagación de dichas referencias se llevó a cabo en la plataforma de Google Académico en correlación con "planeación estratégica y organizaciones" que dio paso a la recolección de las 9 referencias más relevantes que profundizaban y se relacionaban de alguna manera con los objetivos de este artículo.

En este sentido, la elaboración del presente artículo responde en rasgos ordinarios al tema de la planificación estratégica, como herramienta en las empresas ecuatorianas ya que, ésta nos provee de las pautas necesarias para la toma de decisiones acertadas, ubicándose desde la perspectiva empresarial que a su vez deben de ir relacionadas con los objetivos que las instituciones buscan darle alcance y cumplimiento ya sea a corto o mediano plazo.

Sin un plan, no se puede llevar a cabo la correcta organización y administración como lo es la de los recursos financieros, tecnológicos, talento humano, etc., al igual que no se tiene una visión clara de que es lo que se desea conseguir, además de no saber cómo dirigir causando cierto grado de incertidumbre, el no contar con un plan genera que haya pocas probabilidades de conseguir las metas o si se está yendo por el camino correcto, debido a que los planes inexistentes o erróneos alteran el bienestar de la organización.

Por esta razón se destaca que en la actualidad el éxito o fracaso de una empresa está ligado, con que cuenten con una línea a seguir o directrices estratégicas, que ayuden a desarrollarse en todo su potencial, las empresas exitosas son aquellas inquietas que desarrollan sus capacidades competitivas al máximo en el mercado que es cada vez más competitivo, ser competitivos es la fórmula para que una organización se sostenga en el tiempo.

Pero, aquellas organizaciones que no le dan relevancia a la planificación estratégica, se ven vulnerables ante su entorno competitivo por ende no suelen perdurar en el tiempo y aquellas que logren sostenerse están en condiciones inestables en vías de desaparición debido a la falta de una directriz o camino a seguir, causando estancamiento en la misma y tornándose complicado la toma de decisiones y por ende la pérdida de su participación en el mercado.

De modo que las organizaciones deben de establecer hacia donde quieren llegar, siendo una de las ventajas el contar con una planificación estratégica, así como el identificar los recursos con los que cuenta, además de fijarse metas y objetivos que sirven como una guía a seguir para que la organización no pierda su razón de ser, conociendo el mercado en el que se maneja y tener en claro que es lo que necesita para ser competitivos frente a la competencia, anticipándose a los cambios del mercado para que de esta manera se logre sostener en el tiempo en continua preparación y desarrollo.

\section{DESARROLLO}

La estrategia consiste el planteamiento de los objetivos básicos a largo plazo de una entidad y la ayuda de los cursos de acción y la retribución de los recursos precisos para su cumplimento (Capriotti, 1999). Si bien en cierto la planeación es la primera fase dentro de un proceso 
José Peñafiel Loor, Lucy Yulexi Muñiz Soledispa, Luis Fernando Mosquera Tuárez

administrativo y esta figura el desarrollo soberanamente de su tamaño. Para que una empresa alcance el éxito establecido es importante que la persona que emprenda o que ya tenga su negocio conozca el significado e importancia de la planeación, caso contrario pueden surgir problemas y no tomar una decisión correcta para determinar sus ventajas competitivas que aporten a los objetivos señalados de la organización.

Según (Roche, 2007) señala la planeación es aquella que se realiza para anticiparse o aproximarse al futuro en el presente, para poder prepararse y formar escenarios que ayuden a dar forma a las ideas u objetivos. También otros autores destacan que; la planificación se la puede encasillar como una ciencia, la cual al ser aplicada abre paso al establecer metas y objetivos para su respectivo cumplimiento, siendo esta la idónea para la dirigir.

Según (Barranco, 1993), la planificación estratégica es aquel proceso que aborda le abandonar o reafirmar un diferente campo de negocio; al mismo tiempo que ayuda a determinar los recursos disponibles, las diferentes posibilidades de desarrollo o de diversificación, identificación del mercado además de trazar los objetivos a cumplir. Por lo tanto, la planificación es aquella herramienta que sirve para anticiparse al futuro estando en el presente, ayudando a direccionar, así como el de trazar metas y objetivos que ayuden a ser competitivos y se cumpla un fin, así como el de saber con los recursos con los que se cuenta y el cómo usarlos para darle cumplimiento a los objetivos.

Para (Güell, 2019, la estrategia es utilizada de manera habitual para referirse en diversidades de argumentos, al principio esta se la utilizaba para términos militares y en la diplomacia hace aproximadamente medio siglo. Con el pasar del tiempo se ha empezado a generar nuevos términos e interpretaciones, todos son válidos por caber dentro de la generalidad, por lo tanto, podríamos decir que son una serie de eventos llevados acabos para cumplir un fin.

\section{Planeación Estratégica}

Según (Ander, 2007), la Planeación Estratégica es aquella definida como: el conjunto de procesos llevados a cabo dentro de una organización y en su entorno o mercado al que pertenece, esta parte de los objetivos fijados estos pueden ser a corto plazo, mediano o largo, para después seguir una serie de eventos u estrategias para darle cumplimiento a los objetivos previamente establecidos siguiendo directrices que permiten darle la razón de ser de la empresa.

Para (Barrios, 2011), la planeación estratégica seria tiene sus tipologías actuales conforme esta fue encajada de manera inicial en algunas organizaciones de comercio a mediados del año 1950. En aquel entonces aquellas empresas consideradas como importantes fueron quienes fomentaron el desarrollo de sistemas de planificación estratégica de manera seria o formal, nombrados técnicas de planeación de largo plazo.

En este contexto, (CRESPO, 2016) plantea que en el transcurso de la época de los sesenta, la planeación a largo plazo y su término en si prevalecieron en boca de los diferentes especialistas en este campo, desde entonces también se le nombrado por su sinónimo tales como; planeación directiva completa, planeación corporativa completa, planeación general total entre otros 
términos hasta desembocar en el término que se maneja a nivel mundial como lo es la planeación estratégica.

Si bien es conocido que los buenos administradores han formulado buenas estrategias, a esta no se había dado el reconocimiento que la determinase como un factor clave, sino que fueron los estudiosos en la administración que la reconocieron como tal, este reconocimiento tardío se correspondió especialmente a las variaciones del medio ambiente o entorno suscitados a partir de la segunda guerra mundial.

Según (Martínez, 2007), los sistemas de planeación estratégica en el transcurso del tiempo se han mejorado a tal punto que en su actualidad aquellas compañías de renombres e importantes en la economía mundial tienen su propio tipo de sistema, que sirven de ejemplo para las Pymes quienes buscan seguir el mismo camino. De igual manera, (Armijo \& Pública, 2009) plantean que en la actualidad, las empresas reconocen la importancia de la planificación estratégica para su desarrollo y crecimiento a largo plazo.

Si bien se ha demostrado que aquellos gerentes capaces de definir de manera eficiente la misión de la empresa lograran que esta se direccione en mejores condiciones y esté orientada hacia su actividad, ya que las empresas marchan de mejor manera a causa de ello y se vuelven más sensitivas al cambio constante del mercado u entorno. Para (Romero, 2010), las estrategias empleadas en las organizaciones deben estar centralizadas en direccionar las acciones a tomar de manera efectiva para la obtención de resultados insuperables, que doten a la empresa de la capacidad necesaria para solventar sus problemas, generando oportunidades de negocios, para lograrlo se debe de contar con el compromiso y responsabilidad de los colaboradores.

La planeación estratégica está estimada como un instrumento de gran dominio, para el diagnóstico, análisis, reflexión y escoger decisiones para la toma colectiva, acorde al ambiente actual, que servirá de guía o partida para generar el camino a seguir en el futuro, para la generación de cambios y escenarios que el entorno presente, con el fin de conseguir la eficiencia y calidad en su máximo esplendor acorde a las operaciones.

\section{La formulación de la Planificación Estratégica}

Según (Fernández, 2007) "Es una manera de echar un vistazo, examinar y estudiar la real perspectiva competitiva y dificultades estratégicas que estén perturbando a la compañía”. Una culta formulación de la planificación estratégica suministra del contenido para el progreso corporativo mediante la enunciación de la actual posición competitiva y de mercado de la empresa. Asimismo, sirve para la exploración del portafolio transcendental de las diferentes unidades de negocio adentro de la compañía.

Por lo consiguiente (Manes, 2005), define que para implementar una estrategia, se debe inspeccionar la Habilidad de Mercados, el modelo operativo y la distribución organizacional. Estos tres compendios primordiales pertenecen a la pirámide de valor por lo cual podemos distribuir a los diligentes hacia una perspectiva estratégica que haya acogido la compañía. 
En este sentido, (Schwartz \& Vásquez, 2011) aportan que la formulación de la Planificación Estratégica es lucrativa para valorar las expectativas para desarrollarse, emparejar aéreas explícitas para perfeccionar el performance de la definición y ejecución de la estrategia justo entre el portafolio de actividades comerciales presentes e inversiones establecidas. Por ende, la Formulación de la Planificación Estratégica es esencialmente efectiva desde el inicio de un punto de vista externo se consigue proporcionar de ideas y aspectos autónomos sobre inconvenientes y retos que la empresa hoy afronta o afrontara en el futuro.

La figura 1, muestra algunas de las limitaciones que supone, implementar la planeación estratégica en una organización.

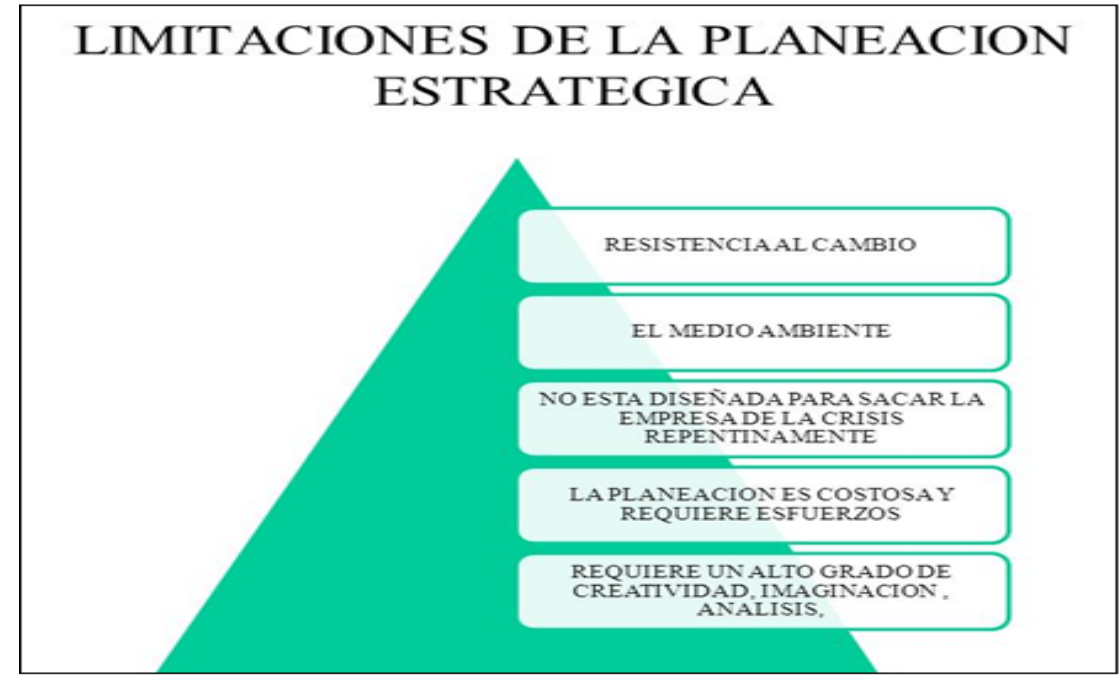

Figura 1. Limitaciones de la planeación estratégica.

Fuente: Tomado de (Elizalde \& Hevia, 2003)

Elaborado por: Autores

La planeación estratégica muestra las siguientes características internamente de una organización (Albavera, 2003):

- Propicia el progreso de la compañía al instituir metodologías de manejo racional de los recursos.

- Minimiza los niveles de indecisión que se pueden mostrar en el futuro, pero no los elimina.

- Tomar medidas en la compañía para hacer frente a las contingencias que se exterioricen, con las mayores garantías de triunfo.

- Conserva una cognición futurista teniendo más orientación del porvenir y un afán de obtener y perfeccionar las cosas.

- Concierta a la empresa en el entorno que lo rodea.

- Instituye un método legítimo para la respectiva toma de decisiones, impidiendo los presentimientos o empirismo.

110 UNESUM-Ciencias. Publicación cuatrimestral. Vol. 4, No. 4 (Septiembre-Diciembre), Año 2020. 
- Minimiza al pequeño los riesgos y aprovecha al total las oportunidades.

- Las decisiones efectuadas se fundan en hechos y no en emociones.

\section{Del entorno donde se desarrollan}

Según los autores (Burgwal \& Cuéllar, 1999)

"Se estima por lo consiguiente que el entorno donde se desarrollan las organizaciones en lo actual por su complejidad, dinamicidad, y variedad precisa de un seguimiento permanente a la grande cantidad de cambios y complicaciones que afronta. Constantemente este desarrolla, se apropia, se convierte se transforma en competitivo por excelentísimo y en ciertos casos fracasa”. Por lo cual queda demostrado que la atribución notable de la decisión estratégica en los efectos ordinarios de cualquier organización.

Así mismo (León, Rivera, \& Nariño, 2009), plantea que la mayoría de las grandes empresas realizan planificación estratégica, mientras que pocas de las microempresas y PYMES dan la razón de utilizarla. Se estima que existe una mayor incidencia de empresas que no dar el brazo a torcer contar con una planificación estratégica, por lo que se puede derivar que existe una cultura gerencial más aguda para una mayor exigencia de parte de empresas y establecimientos financieros, gubernativos, agencias internacionales etc.

Las primordiales razones por las que las compañías no manipulan este instrumento de gestión, conciernen a características de tamaño, la actitud del administrador, inexperiencia, y por otros elementos con transcendental significado para no planificar.

\section{Modelos de planeación estratégica más conocidos}

El Balanced Scorecard o Cuadro de Mando Integral, es un modelo de planificación estratégica que permite visualizar desde cuatro perspectivas distintas para organizase en cuanto a objetivos e iniciativas. La figura 2, muestra las perspectivas propuestas por el modelo. 


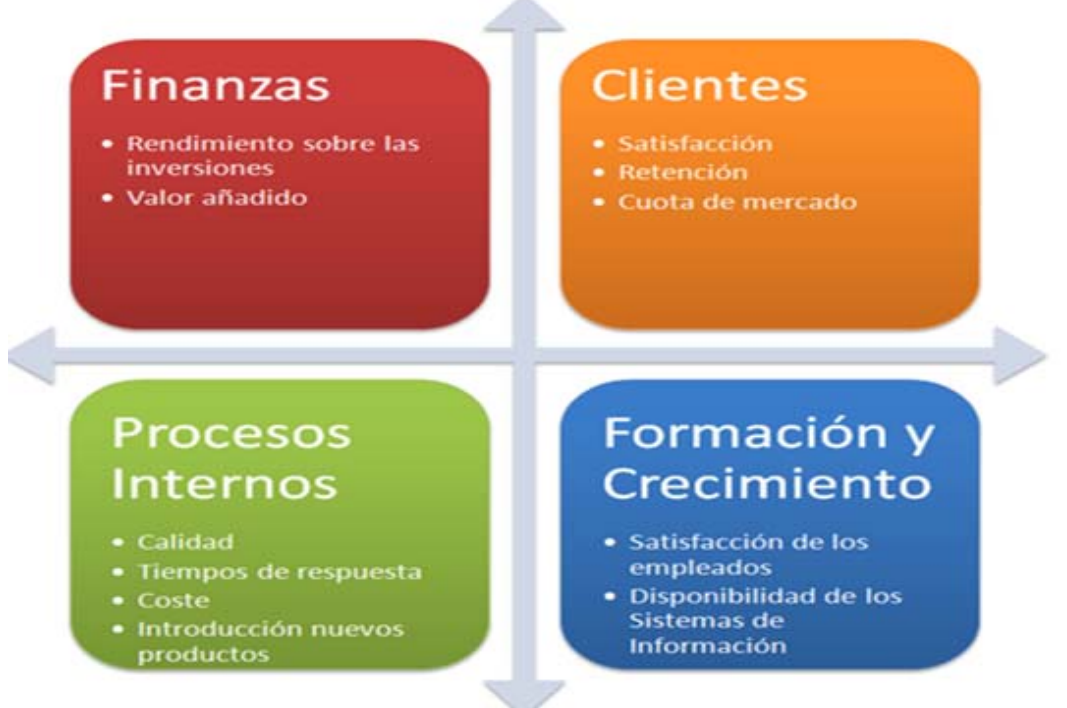

Figura 2. Balanced Scorecard o Cuadro de Mando Integral. Fuente: GESTIÓN PENSEMOS

Elaborado por: Autores

La figura 3 muestra el modelo llamado Mapa estratégico. Este modelo resulta útil para comunicar la estrategia a la organización y su entorno, no resulta ser un modelo de plan estratégico en sí, pero resulta útil para evitar la incertidumbre ya que se puede aplicar en conjunto con un modelo estratégico.

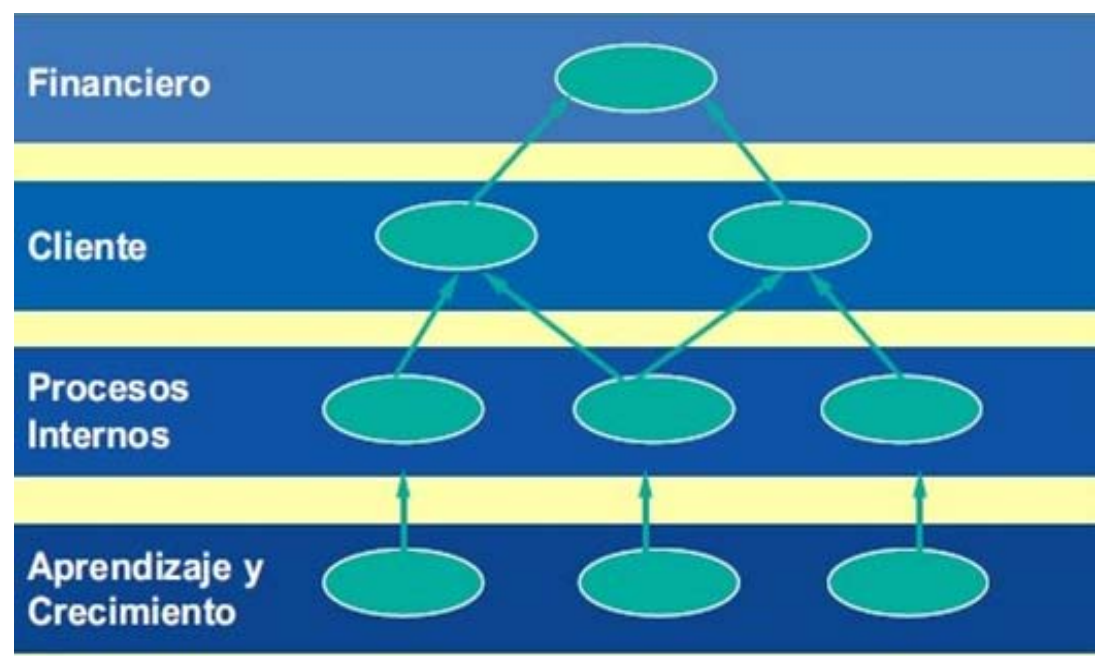

Figura 3. Mapa estratégico.

Fuente: GESTIÓN PENSEMOS

Elaborado por: Autores

La figura 4 muestra el modelo Las 5 Fuerzas de Porter. Este es un modelo estratégico que resulta útil para poder determinar el grado de influencia y competencia que tiene la empresa dentro de un mercado, sirve mucho para lograr establecer una estrategia de negocio.

112 UNESUM-Ciencias. Publicación cuatrimestral. Vol. 4, No. 4 (Septiembre-Diciembre), Año 2020. 


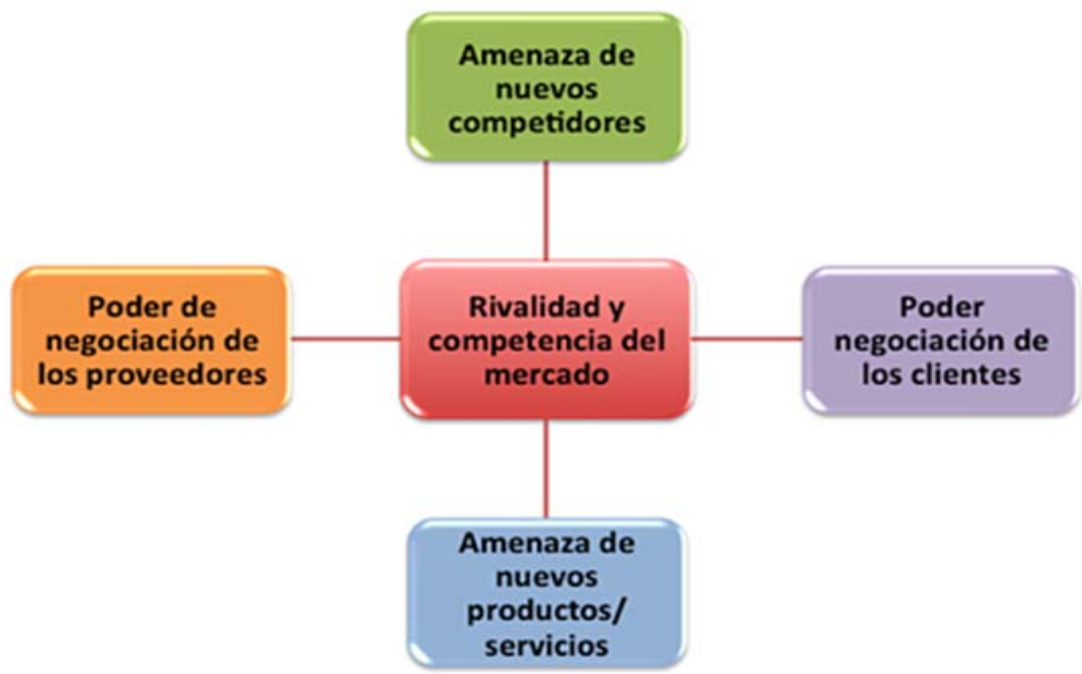

Figura 4. Las 5 Fuerzas de Porter.

Fuente: GESTIÓN PENSEMOS

Elaborado por: Autores

La figura 5 muestra el modelo FODA. Este quizás sea el modelo de planificación estratégica más conocido, debido a su capacidad para poder ser adaptado a cualquier organización, pues este permite diagnosticar la situación real que vive la empresa para poder plantear estrategias a futuro.

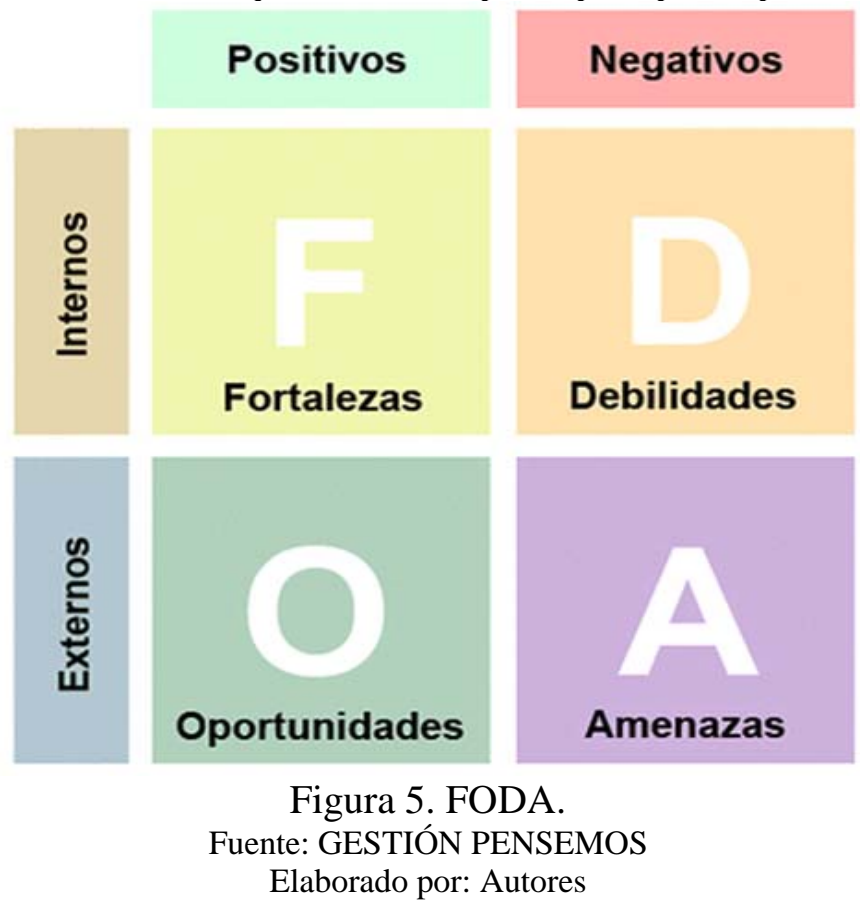

\section{Ventajas de la planificación estratégica}


- Apoya a acciones sistemáticas y con un fin.

- Marca necesidad de cambios a futuros.

- Suministra una base para la inspección.

- Exige a la visualización de un todo.

- Rige la vigilancia hacia los objetivos.

\section{Desventajas de la planificación estratégica}

- Limitación por la insuficiente exactitud de información y por la inseguridad de los hechos futuros.

- La planeación tiene un costoso precio.

- La proyección obtiene "ahogar” la iniciativa.

- Consigue retardar en cualquier caso las operaciones

\section{RESULTADOS}

De acuerdo a lo investigado y los métodos utilizados se puede definir que desde tiempos remotos la planificación estratégica es transcendental por distintos aspectos ya que suministra un marco de referencia para la actividad organizacional dentro de la empresa, que pueda llevar a un mejor trabajo y una mayor armonía de la organización, porque si no se tiene planes los administradores no poseerían una idea clara de lo que se requiere organizar, por ende en el $90 \%$ de las empresas mediana y grandes es primordial y vital tener una meta, un objetivo ya establecido para tomar las respectivas decisiones en la entidad, y el 95\% en relación a las microempresas unifamiliares y familiares que por ser emergentes o casuales existe una planificación estratégica empírica

Es sustancial indicar que de la investigación realizada a las empresas y microempresas tienen realidades distintas; a las primeras se torna una herramienta elemental realizar las planificación estratégica que es a largo plazo (5 años), para de ahí plasmar la planificación anual en forma prospectiva para alcanzar los resultados propuestos a largo plazo, no así las microempresas, cuya debilidad fundamental radical en el desconocimiento de una planificación estratégica que proyecte el desarrollo de la misma a largo plazo, debido a que su situación general es conseguir resultados óptimos inmediatos para satisfacer ingresos económicos carentes en la familia

Dentro del contexto del conocimiento e importancia de la planificación estratégica como ciencia el $100 \%$ de la población de estudio sabe de su importancia como herramienta gerencial y de crecimiento empresarial. No está de más sintetizar que la planificación estratégica permite medir a través de indicadores los resultados alcanzados y asi tomar decisiones gerenciales que les permite rectificar o mejorar su desenvolvimiento, para ajustarse a los cambios y a las instancias que les atribuye el ambiente y adquirir el máximo de eficiencia y calidad de sus prestaciones.

De esta manera la planeación estratégica como ciencia, es un proceso sistemático, que da sentido de orientación y persistencia a las actividades cotidianas de una organización, admitiéndole imaginar el futuro y asemejando los recursos, principios y valores solicitados para circular a partir del presente hacia el futuro, persiguiendo una serie de procedimiento operativos y administrativos que alcancen la consecución de los objetivos a largo plazo, ayuda a determinar

114 UNESUM-Ciencias. Publicación cuatrimestral. Vol. 4, No. 4 (Septiembre-Diciembre), Año 2020. 
prioridades, accede concentrarse en las fortalezas de la entidad, trata de minimizar los problemas de cambios en el hábitat interno y externo.

La implementación de la planificación estratégica en las empresas y microempresas de acuerdo a los trabajos realizados por terceros, así como aporte a la ciencia por autores como (Burgwal\& Cuéllar, 1999). "Se estima por lo consiguiente que el entorno donde se desarrollan las organizaciones en lo actual por su complejidad, dinamicidad, y variedad precisa de un seguimiento permanente a la grande cantidad de cambios y complicaciones que afronta. Constantemente este desarrolla, se apropia, se convierte se transforma en competitivo por excelentísimo y en ciertos casos fracasa”. Por lo cual queda demostrada la atribución notable de la decisión estratégica en los efectos ordinarios de cualquier organización.

La empresas que aplica la planificación estratégica obtendrá grandes y favorables resultados ya que le permitirá tener tranquilidad, al saber que se está siguiendo al camino del éxito eso es gratificante tanto para el personal como para la empresa, se obtendrá además una buena comunicación con los colaboradores, logrando que se sientan motivados y continuar con el trabajo encomendado. La planificación estratégica es sustancial para las empresas porque acopla las fortalezas productivas con las oportunidades de mercado y ofrece una orientación para efectuar las metas.

\section{CONCLUSIONES}

La planificación estratégica cumple el rol dentro de las organizaciones de guiar y ayudar a que una organización se encamine por un buen camino acorde a sus metas y objetivos establecidos mediante la aplicación sistemática de eventos que ayudan a esta. Los modelos de planificación estratégica son de vital ayuda, en especial los modelos más conocidos debido a que estos ya han sido aplicados por otras organizaciones a las cuales ya les ha funcionado por lo tanto sirven de guía tales como el análisis DAFO y las 5 fuerza de Porter.

Los métodos de planeación estratégica no son tan sencillos de aplicar, ya que esta requiere que sea aplicada por los mandos más altos dentro de una organización que a su vez va a requerir de sus colaboradores y cierto grado de compromiso por lo tanto esta determinara su éxito o frustración, durante su aplicación presentaran desventajas y ventajas, pero siempre será ventajoso aplicar un plan estratégico ya que ayuda a anticiparse al futuro.

\section{REFERENCIAS BIBLIOGRÁFICAS}

Albavera, F. (2003). Planificación estratégica y gestión pública por objetivos. 32. United Nations Publications.

Ander, E. (2007). La planificación estratégica. Buenos Aires: Lumen.

Armijo, M., \& Pública, G. (2009). Manual de planificación estratégica e indicadores de desempeño en el sector público. 103. Ilpes/Cepal.

Barranco, F. (1993). Planificación estratégica de recursos humanos: del marketing interno a la planificación. Pirámide.

Barrios, A. (2011). Planificación estratégica, presupuesto y control de la gestión pública. Universidad Catolica Andres.

Burgwal, G., \& Cuéllar, J. (1999). Planificación estratégica y operativa aplicada a gobiernos locales. Manual de facilitación: incluye materiales para los participantes. Editorial Abya Yala. 
Capriotti, P. (1999). Planificación estratégica de la imagen corporativa.

CRESPO, P. (2016). Planificación estratégica y gestión de la publicidad. . Conectando con el consumidor. ESIC.

Elizalde, A., \& Hevia, A. (2003). Planificación estratégica territorial y políticas públicas para el desarrollo local. 29. United Nations Publications.

Fernández, M. (2007). Planificación Estratégica de las ciudades. Investigaciones regionales: Journal of Regional Research, 10, 269-275.

Güell, M. (2019). Planificación estratégica de ciudades: Nuevos instrumentos y procesos. 10. Reverté.

León, A., Rivera, D., \& Nariño, A. (2009). Relevancia de la gestión por procesos en la planificación estratégica y la mejora continua. 2, 65-72. Eídos.

Manes, J. (2005). Gestión estratégica para instituciones educativas. Ediciones Granica.

Martínez, J. (2007). Metodologías avanzadas para la planificación y mejora. planificación estrategica, BSC. Ediciones Díaz de Santos.

Roche, F. (2007). La planificación estratégica en las organizaciones deportivas (Vol. 26). Editorial Paidotribo.

Romero, A. (2010). Dirección y planificación estratégica en las empresas y organizaciones. Ediciones Díaz de Santos.

Rovere, M. (2006). Planificación estratégica de recursos humanos en salud. edición ampliada y corregida. OPS.

Schwartz, P., \& Vásquez, J. (2011). La planificación estratégica por escenarios. Cuadernos de Administración, 14(21), 199-225.

116 UNESUM-Ciencias. Publicación cuatrimestral. Vol. 4, No. 4 (Septiembre-Diciembre), Año 2020. 